Théologiques

Théologiques

\title{
Le rien et Dieu chez Cioran
}

\section{Marc Dumas}

Volume 4, numéro 2, octobre 1996

\section{L'épreuve du rien}

URI : https://id.erudit.org/iderudit/602441ar

DOI : https://doi.org/10.7202/602441ar

Aller au sommaire du numéro

\section{Éditeur(s)}

Faculté de théologie de l'Université de Montréal

\section{ISSN}

1188-7109 (imprimé)

1492-1413 (numérique)

Découvrir la revue

\section{Citer cet article}

Dumas, M. (1996). Le rien et Dieu chez Cioran. Théologiques, 4(2), 83-98. https://doi.org/10.7202/602441ar

\section{Résumé de l'article}

Cet article explore le monde et la pensée de Cioran sur le Rien. Il dégage tout d'abord l'horizon cioranien, où l'humain semble écartelé entre le Rien originaire, dont il peut à l'occasion faire l'expérience, et le néant de son existence. Devant cela, Cioran lance un appel à une plus grande lucidité, pour éviter les diverses illusions offertes sur le marché de l'existence. L'article présente ensuite diverses compréhensions du Rien. Celui-ci est alors mis en corrélation avec le thème de la mort et celui de Dieu. L'auteur souligne enfin l'apport possible de l'oeuvre cioranienne pour le travail théologique aujourd'hui. 


\title{
Le rien et Dieu chez Cioran
}

\author{
Marc DUMAS \\ Faculté de théologie \\ Université de Sherbrooke
}

\section{RÉSUMÉ}

Cet article explore le monde et la pensée de Cioran sur le Rien. Il dégage tout d'abord l'horizon cioranien, où l'humain semble écartelé entre le Rien originaire, dont il peut à l'occasion faire l'expérience, et le néant de son existence. Devant cela, Cioran lance un appel à une plus grande lucidité, pour éviter les diverses illusions offertes sur le marché de l'existence. L'article présente ensuite diverses compréhensions du Rien. Celui-ci est alors mis en corrélation avec le thème de la mort et celui de Dieu. L'auteur souligne enfin l'apport possible de l'œuvre cioranienne pour le travail théologique aujourd'hui.

This article explores the world and thoughts of Cioran on nothingness. It explains, firstly, the cioranian horizon where the human being seems to be torn between original nothingness, which he can experience on occasion, and the nothingness of his existence. Facing this, Cioran appeals to a greater lucidity to avoid the illusions offered by the market of existence. Secondly, this article presents diverse understandings of nothingness. This is then correlated with the theme of death and that of God. The author underscores the contribution of cioranian works for contemporary theology.

Lorsque nous avons englouti le monde et que nous restons seuls, fiers de notre exploit, Dieu, rival du Rien, apparaît comme une dernière tentation. (Des larmes et des saints, p. 295)

Je suis [...], un ci-devant Rien et sans même le privilège d'avoir jamais pourri. (Précis de décomposition, p. 698)

Sans Dieu tout est néant; et Dieu? Néant suprême. (Syllogismes de l'amertume, p. 777) 
Qui connaît ne serait-ce que quelques fragments ou quelques titres de l'œuvre de Cioran ne s'étonnera pas de ce choix pour approfondir le thème du rien. On peut par contre se demander comment et pourquoi réunir Dieu et Cioran. N'est-il pas tout sauf un croyant ou un théologien? J'ai au moins deux raisons pour opérer ce curieux rapprochement. La première, c'est que l'on parle souvent de Cioran comme d'une autorité capable de mettre en pièces le travail théologique. Certes, Cioran dénonce et critique sévèrement les théologiens, parce qu'ils sont essentiellement incapables d'exprimer le réel. Incapables de se dépêtrer des illusions qui les aveuglent, ils tiennent trop peu compte de l'existence réelle trempée dans le mal, de cet alliage d'être et de non-être. Et s'ils ne plongent jamais au cœur du mal, ces collaborateurs de l'illusion ne font que jongler avec des mots insensés. De plus, ces fonctionnaires incroyants ne réussissent pas à traiter avec expérience de Dieu. La théologie est inapte à exprimer l'essentiel : l'absolu.

La seconde raison que j'avance pour rapprocher l'écrivain d'origine roumaine et le sujet du travail théologique (Dieu), c'est que Cioran parle très souvent de Dieu. Je ne suis d'ailleurs pas le premier à insister sur cette dimension de son ouvre. Plusieurs notent l'importance relative du religieux, de la divinité, de la recherche spirituelle, etc. dans son cuvre ${ }^{1}$. Sylvie Jaudeau publiait par exemple en 1990 un essai sur Cioran intitulé Cioran ou le dernier homme ${ }^{2}$. Elle présente Cioran ultimement comme un gnostique, qui propose une voie radicale de renoncement et comme un mystique ayant "une soif d'absolu en son état nu, sauvage, réduite à son essence et délestée des entraves du sacré ${ }^{3}$ ». Voilà pourquoi il est opportun d'examiner l'ouvre de Cioran: elle se rapporte à l'activité théologique et au propos de notre essai : le Rien.

Je ne cherche pas ici à commenter savamment Cioran, mais plutôt à le rencontrer. Et les quelques centaines de pages que j'ai lues attentivement me donnent simplement la possibilité de brosser un tableau de sa lecture du monde et de sa compréhension du Rien. L'exercice ne sera pas vain dans la mesure où nous découvrirons une voix décrivant ce que vivent plusieurs de nos contemporains. Mais avant de présenter l'horizon de Cioran, d'approfondir la thématique du Rien proprement dite et de

1 Voir J. N. VUARNET, « L'école du vertige ", dans Magazine littéraire, déc. 1994, p. 35-38.

2 Sylvie Jaudeau, Cioran ou le dernier homme. Paris, José Corti, 1990. Voir aussi : Sylvie Jaudeau, Entretiens. Paris, José Corti, 1990.

3 Ibid., p. 108. 
proposer une lecture théologique, donnons quelques brefs repères bio- et bibliographiques. Qui est Cioran? Pourquoi et qu'écrit-il?

Il n'est pas facile de qualifier l'homme Cioran. Était-il athée? Nihiliste? Pessimiste? Crois pas. Etait-il écrivain? Philosophe? Sceptique? Peut-être. Était-il un penseur? Un éveillé? Un chercheur d'absolu? Probablement. Émil Cioran, né en Transylvanie en 1911, a vécu presque soixante années de sa vie à Paris, avant de s'éteindre en juin 1995. Étudiant en philosophie à Bucarest, il ira en 1937 à Paris pour réaliser une thèse sur Bergson qu'il ne terminera jamais. Il laisse une œuvre que Gallimard vient de publier dans sa collection quarto ${ }^{4}$. Presque mille huit cents pages rassemblant la majeure partie de sa production tant roumaine que française. La lecture de Cioran produit tantôt un effet décapant sur son lecteur; tantôt elle entraîne chez ce dernier sourire et admiration, tant le style translucide est enlevant.

Homme hanté par l'absolu, illuminé le temps de l'éclair, foudroyé pour l'éternité de devoir ne pas y être, Cioran s'ennuie dans le temps et s'imagine pouvoir le liquider. Il dénonce toute pensée qui s'installe dans ce temps, qui s'investit dans toutes les formes d'illusion, soit religieuse ou non, soit sage ou non. Il divague vers l'autre rive, celle de l'absolu. Tout en étant écœuré de la superficialité qui l'entoure et qui le tourmente profondément aussi en lui-même, il se voit comme impuissant à repousser les apparences, à dépasser les pseudo-absolus. Et c'est dans cette impuissance qu'il s'engage. Ce désengagement, cette neutralité ouvre-t-elle une voie à l'absolu? Cette tentation d'absolu apparaît chez Cioran comme un souvenir indicible, impossible à atteindre aujourd'hui. Cioran, un quêteur d'absolu, qui décrie la vanité de sa quête?

Pourquoi alors écrire et écrire? Ne serait-il pas suffisant de lancer un immense cri de désespoir? Ou bien d'écrire un réquisitoire et de se taire? Cioran lui-même s'en veut d'en faire trop. Mais ne prend-t-il pas un malin plaisir à multiplier les fragments et les entretiens? Écrire et autant parler ne risque-t-il pas d'invalider la pensée, d'évider le projet de son contenu? L'écriture est grisante, les mots recherchés, les expressions crues et incisives. Pour quelqu'un qui cherche à se déprendre du néant et à se fondre avec le Rien...

Mais que désire l'indésiré devant l'indésirable? Pourquoi se faire délateur de la quotidienneté, épiscope du vécu quotidien? Et ces centaines de pages explicitent-elles la plainte, le hurlement tantôt apeurant, tantôt dérangeant de Cioran? Des centaines de fragments reprennent 
longuement sous toutes ses coutures le mal-être de l'homme. Ils gravitent autour de trois pôles principaux. Le premier dénonce les travers de l'existence qui camouflent l'essentiel et renforcent les mensonges des hommes, les pseudo-vérités qu'ils gobent devant la vie. Le second dénonce toute petite vie bien rangée, coupée de la source chaotique de la vie. Cette source tarie, l'homme vit bêtement et platement; il s'imagine d'autres possibles au lieu de voir qu'il est tiré du Rien. Il en veut à l'esprit ou à la conscience, cette étincelle de décadence, qui conduit à une vie signifiante. Leurre terrible pour Cioran! Démasquer la vie et l'accepter vraiment comme elle est : un mélange d'être et de non-être. Et le dernier pôle s'intéresse à ceux qui décrochent de ce cruel réel, soit parce qu'ils se sacrifient eux-mêmes, soit parce qu'ils sont sacrifiés par le Rien.

Avant d'approfondir la pensée cioranienne à propos du rien, présentons très brièvement les cinq œuvres lues. Elles furent publiées entre 1932 et 1973 et manifestent toutes un intérêt pour la constellation du néant, du rien, du vide, du non-être, de la vacuité. Le premier écrit publié en roumain en 1932 contient en germe ce qui sera sans cesse repris plus tard. Sur les cimes du désespoir est déjà constitué de fragments, qui martèlent des thèmes comme ceux de la vie et de la mort, de l'esprit et de la folie, de l'absurde, de la mélancolie, du désespoir, etc. C'est à cette époque qu'exténué par des veilles ininterrompues, il se rend compte de l'inanité de la philosophie ${ }^{5}$. Écrire ce livre dans ce contexte est pour lui une "sorte de libération, d'explosion salutaire. Si je ne l'avais pas écrit, j'aurais sûrement mis un terme à mes nuits ${ }^{6}$ ". Puis, Des larmes et des saints, publié en 1937 peu de temps après son départ pour Paris, est un livre qui "déroute et fait scandale ${ }^{7}$ ». Sévèrement critiqué pour son nihilisme et sa confusion, les fragments de cet opuscule ressassent tous les éléments d'une crise religieuse profonde: Dieu, le monde et son néant, le Rien, les larmes et les saints, etc. Précis de décomposition, le premier ouvrage rédigé en français, fut publié en 1949. Les fragments, regroupés en sections intitulées, mentionnent régulièrement les termes de notre constellation: le rien, le néant, le vide, l'inanité,... La tentation d'exister et De l'inconvénient d'être né, œuvres parues respectivement en 1956 pour la première et en 1973 pour la seconde, sont deux autres textes auxquels nous nous sommes intéressés. Ils sont plus tardifs, mais insistent sur les constellations et les conjuguent de façon quelque peu différente pour notre profit.

5 Voir Emil CIORAN, « Sur les cimes du désespoir », dans CEurres, p. 17.

$6 \quad$ Idem.

7 E. MARENCO, "Repères biographiques ", dans Magazine Littéraire, déc. 1994, p. 28. 


\section{L'horizon de Cioran}

Si j'ai affirmé plus haut que Cioran interpelle le théologien d'aujourd'hui, c'est parce que cet auteur se meut sur un horizon dialectique complexe et difficile à intégrer. Le réel, dont il rend compte, contrarie radicalement la lecture traditionnelle développée par la théologie. Ce choc des horizons nous provoque et nous lance un défi, non seulement à cause du déséquilibre engendré, mais aussi à cause de l'ouverture qu'il nécessite pour rencontrer celui qui pense et est autrement. Je ne cherche donc pas ici à récupérer en notre faveur l'œuvre littéraire de Cioran. Il est loin le temps où on se servait de la littérature en théologie comme d'une source potentielle de citations en faveur ou contre les résultats du travail théologique. Le théologien apprend de plus en plus, et j'espère de mieux en mieux, à lire et à respecter ce que l'écrivain exprime. Mais n'y a-t-il pas un gouffre infranchissable entre ces deux pics aux versants abruptes et mortels? Comment, en effet, celui qui croit et tente de rendre compte de sa foi et de son espérance en Christ peut-il converser avec celui qui souhaite un retour non pas simplement à l'origine, mais encore à l'inexistence même?

Ce qui intéresse le théologien, ne serait-ce pas justement de gravir l'autre sommet pour découvrir la critique des illusions et la recherche de l'absolu de celui qui se pose autrement dans ce monde? Ne serait-ce pas de découvrir aux détours des mots, si leur emballement ne cerne pas autrement le Dieu au-delà de Dieu, le Vide, le Rien? Nous osons l'aventure du fragment, la lourdeur accablante et angoissante des mots de l'écrivain, pour voir ce que nous pouvons apprendre à propos de Dieu et du Rien. La tentation de glisser vers une manière de faire plus proche de Cioran existe : délaisser l'effort de synthèse et décrire existentiellement l'écartèlement entre ceci ou cela ou encore proposer telle non-voie d'être, etc. Je résiste pourtant à imiter ce qui n'est pas mien et, quoique encore sous le choc des lectures de Cioran, j'évite l'emprunt d'une forme plus fragmentée ou plus aphoristique.

L'horizon de Cioran est un horizon mouvant marqué par plusieurs éléments, dont les principaux m'apparaissent être les suivants : il insiste tout d'abord fortement sur la puissance initiale du Rien, qui soutient l'existence et fait parfois irruption dans l'existence, puis sur le néant de l'expérience du réel et enfin, sur la lucidité à exercer face aux illusions possibles concernant le réel. Présentons brièvement ces éléments avant d'approfondir sa compréhension du Rien. 


\subsection{Un horizon marqué par la puissance initiale du Rien}

La vie est une échappée du chaos primordial. En soi, elle est créatrice et destructrice; elle est turbulente, insupportable, voire démentielle. Voilà pourquoi Cioran se fascine principalement pour les premiers et les derniers instants de la vie, ceux qui représentent son irruption et son effondrement ${ }^{8}$. La transition entre la non-existence et l'existence et celle entre la vie et la mort sont pour lui fondamentales, car elles laissent les traces de ce chaos primordial. Là où le temps est absent ou suspendu (ceci peut aussi arriver à l'intérieur du temps, c'est l'extase, la présence de l'éternel maintenant), il est possible de goûter le sublime, l'extase, l'enthousiasme, l'éternité ou, au contraire, de se tenir dans le chaos, l'anarchie, la contradiction,... L'entre-deux, le temps, la durée, la vie ordinaire, l'existence, le réel sont marqués profondément par cette origine démentielle, mais semblent le plus souvent incapables d'en vivre. Cioran, s'il s'intéresse aux transitions, ne néglige point les moments extrêmes dans l'existence où s'accomplit une "conversion au Rien ${ }^{9}$ ». Qu'est-ce à dire?

Ceci implique tout d'abord qu'il existe à travers la quotidienneté, au niveau du vécu, des instants ou des expériences sommets. Ces moments surviennent comme un raz-de-marée qui submerge, engloutit et "projette tout droit dans le néant». Ce «tourbillon sauvage " de la vie est d'une intensité telle, d'une telle exhubérance et d'une telle plénitude, qu'il nous montre le vide et l'absurde du quotidien vécu. Ces instants vertigineux, où les forces vitales incontrôlables nous subjuguent, nous déconcertent l'instant d'après; vivre est alors quasiment insupportable, tant tout nous semble illusoire. En plus du caractère incontrôlable de la vie qui se manifeste lors d'expérience-limite, vivre intensément ou radicalement, c'est-àdire en connexion avec le bouillon chaotique de la vie, contient un certain risque parce que l'ordinaire n'y résiste pas. La vie ordinaire, sauve de la vie extrême, insouciante quant à sa dimension essentielle de profondeur, est une vie aplatie, une "p'tite vie ". Mais d'avoir touché la radicalité de la vie conduit à une dévitalisation de la vie ordinaire; elle est néant et rien comparée à l'expérience sommet. Vivre intensément comporte donc un risque, car on ne peut ensuite que se demander comment continuer de vivre une vie ordinaire. Le néant de l'ordinaire face au Rien radical, voilà une distinction qu'il nous faudra préciser plus loin.

Le Rien avec la majuscule apparaît premièrement comme un présupposé chez Cioran. Dans certains fragments, il est le principe chaotique

8 Voir Emil ClORAN, "Précis de décomposition », dans $E_{\text {Eures, p. } 690 .}$

9 Voir Emil CIORAN, «Sur les cimes du désespoir ", dans CEurres, p. 22-23. 
primordial, alors que dans d'autres, il est comme un possible aboutissement du processus de néantisation ou un concept-limite. Ailleurs encore, le Rien est comme une faille au cœur de l'existence; comme une interruption de l'existence, il apparaît à celui qui est saisi, ravi, enlevé lors de cette expérience extraordinaire.

\subsection{Un horizon marqué par le néant de l'existence}

Certains fragments de Cioran décriront cette chute dans l'existence ou encore cette retombée dans l'existence, une fois l'expérience sommet passée. D'autres s'attaqueront aux affres de l'existence baignée dans le néant. Les réflexions parfois abstraites de Cioran reflètent le plus souvent ses observations du réel. Ses fragments s'accrochent directement au réel, au sien et à celui des autres. L'écrivain réagit par conséquent sévèrement contre ceux qui nomment, formulent, définissent et réduisent la vie en systèmes, alors qu'elle est originairement touffue, éclatée, féconde, chaotique. D'ailleurs ceux qui parlent autrement du néant qu'en termes d'affectiviré, perdent leur temps ${ }^{10}$.

En décrivant des rencontres, des observations, des réactions et tout ce qu'il peut passer au peigne du scepticisme, Cioran ne se fait-il pas, d'une certaine façon, le porte-étendard de plusieurs d'entre nous qui ne vivent pas, mais vivotent? Ces derniers retrouvent en lui une éminence grise qui broie du noir tout comme eux en bavent. Exilés et exclus des sociétés, ils n'accéderont à rien. Cioran décrit la réalité des déclassés et des désolidarisés, une réalité encrassée dans le cambouis de la mortitude. Mortels, ils n'espèrent plus rien, car ils vivent de néant en néant. Cioran vit et décrit ces vies, ces anti-vies selon les bien-pensants. Elles nous révèlent durement la réalité. Plus moyen de cacher les inconsistances du réel. Plus moyen de camoufler ces ratés. Plus moyen d'avoir une bonne nouvelle pour ces désillusionnés, ces néantisés, ces moins que rien. Ces descriptions de la nullité et de la stérilité coïncident avec son appel à rompre avec la normalité pour rebrousser chemin vers la vie chaotique. Ce bouleversement, cette désintégration, marque l'« apothéose du non-être, le triomphe du Rien ${ }^{11} \gg$. Mais pourquoi alors être, exister et vivre? Tout semble n'être qu'un faux pas:

Par quel artifice ce qui semble être se dérobe au contrôle de ce qui n'est pas? Un moment d'inattention, d'infirmité au sein du Rien : les larves en profitèrent; une lacune dans sa vigilance : et nous voilà. Et de même que la vie supplanta le néant, elle fut supplantée, à son tour, 
par l'histoire: l'existence s'engagea ainsi dans un cycle d'hérésies qui minèrent l'orthodoxie du néant 12 .

Cette citation, tirée du Précis de décomposition, illustre assez bien la compréhension du réel pour Cioran. La vie n'origine d'aucune volonté; elle est une lacune, une erreur, une escapade malencontreuse au sein du Rien. C'est pour cette raison que Cioran ne pose, ni ne présuppose de sens à la vie. Mais pourquoi vivre? Pourquoi ne pas en finir avec la vie? Un court fragment intitulé Rien n'a d'importance tient presque de la confession lorsque Cioran avoue ne pas savoir pourquoi il vit, ni pourquoi il ne cesse pas de vivre. Au lieu d'exister dans le monde, il préfèrerait " planer tout seul dans le néant ". La vie est irrationnelle; elle est là sans raison, sans intérêt. Pourquoi donc s'interroger et essayer de trouver un sens à la vie? Tout paraît vide et stérile; même l'expérience du néant lui semble illusion ${ }^{13}$. Ailleurs, pourtant, il laisse entrevoir pourquoi il ne se tue pas:

C'est que [...] le rien qui fait prolonger les actes est pourtant d'une force supérieure à tous les absolus; il explique la coalition tacite des mortels contre la mort; il est non seulement le symbole de l'existence, mais l'existence même; il est le tout. Et ce rien, ce tout ne peut donner un sens à la vie, mais il la fait néanmoins persévérer dans ce qu'elle est : un état de non-suicide ${ }^{14}$.

Ce rien, cette existence perdure dans le réel et ne cherche pas à s'éteindre volontairement.

\subsection{Un horizon marqué par la lucidité}

Depuis la flânerie jusqu'au carnage, l'homme ne parcourt la gamme des actes que parce qu'il n'en perçoit point le non-sens : tout ce qui se fait sur terre émane d'une illusion de plénitude dans le vide, d'un mystère du Rien... En dehors de la Création et de la Destruction du monde, toutes les entreprises sont pareillement nulles ${ }^{15}$.

L'horizon de Cioran est tout d'abord marqué par le Rien de l'origine et par le néant de l'existence. Un autre élément important chez Cioran est le devoir qu'il se fait de placer son lecteur devant la nudité du réel et de l'existence. Tant mieux s'il réussit à donner le vertige à ceux qui s'étaient

12 Emil CIORAN, «Précis de décomposition ", dans Euvres, p. 710

13 Voir Emil CloRAN, "Sur les cimes du désespoir", dans CEuvres, p. 42. Si cette expérience lui semble être une illusion, on comprend pourquoi l'appel à la lucidité est un dépassement de l'expérience du néant et pourquoi elle est irréelle tout comme le Rien.

14 Emil CIORAN, « Précis de décomposition ", dans EEuvres, p. 596.

Ibid., p. 644 . 
refugiés dans les tours d'ivoire; tant mieux s'il permet à son lecteur de percevoir le néant de l'existence. Il ne sera que mieux pour sauter dans le vide du Rien ou mieux pour rêver de l'inaccessible rêve ${ }^{16}$.

Cioran s'attaque tout d'abord à ceux que l'on pourrait qualifier de normaux ${ }^{17}$, c'est-à-dire ceux qui s'illusionnent et ne perçoivent absolument plus la réalité. Il y a, par exemple, les philosophes qui ont troqué le monde de la vie pour celui des idées et des systèmes. Il y a encore les croyants, qui projettent du sens et espèrent un monde meilleur, au lieu de voir le néant de l'existence. Il y a encore tous les autres qui font l'autruche et qui mordent à belles dents dans le fruit de la vie, sans y déceler toute sa pourriture. Ils espèrent, projettent, imaginent et perdent la réalité de vue. Ces gens se tuent à exécrer du sens et de la vie et à rendre compte de l'existence, de cette incartade de la vie anarchique et démonique. Cioran se moque des tentations et des tentatives d'encadrer l'incadrable, de raisonner l'irrationnel. Il critique ceux qui domptent le démonique en eux, c'est-à-dire cette tension créatrice et destructrice fondant ce que nous sommes : originairement chaos, barbares,... Il oppose à l'inconscience et à la naïveté la désespérante lucidité de l'existence.

Mais quelles seraient les meilleures attitudes à adopter pour éviter de tomber dans les pièges de l'illusion? Peut-on résister à la tentation de naître ou d'exister, retrouver l'attitude chaotique, faire une expérience extatique ${ }^{18}$ ou être en quête d'une telle expérience, apprendre à voir et ensuite à vivre la vacuité de la réalité, un peu comme les bouddhistes?

Cioran médite longuement sur l'ante-naissance, sur la pré-existence, sur l'avant la chute ou l'exil dans la durée et le temps. Voilà pourquoi il recueille les fruits de ceux qui disent ou dont on dit qu'ils affirment transcender la durée, l'histoire, bref le temps. Cioran observe, par exemple, attentivement les mystiques de la tradition chrétienne, ces acrobates qui sautent hors du cœur du réel et qui parfois s'abîment dans la lumière du Rien. Ces gaillards n'ont rien des fluets qu'on s'imagine; ils se lançent à

Voir ibid., p. 621. Le saut et le désir sont-ils des expressions adéquates pour caractériser l'intention de Cioran? Sa position semble parfois ambivalente : d'une part il semble incapable de résister à la tentation de faire une expérience mystique et d'autre part elle sera dénoncée comme une illusion, la plus grande qui soit.

17 Notons l'ambivalence de certaines expressions chez Cioran. Si dans certains cas, les gens normaux sont perçus de façon péjorative, ils sont dans d'autres contextes, inversement, perçus positivement, car ils sont ceux qui ne s'illusionnent plus sur le réel et qui savent qu'il est du rien. Les contextes sont alors essentiels pour comprendre les mots de l'auteur.

Comme Cioran le laisse entendre. 
la rencontre d'un possible dépassement de la normalité, « ils violentent le Rien pour l'animer ${ }^{19}$ ».

N'évitons donc pas l'anormalité et gardons notre sang chaud le plus longtemps possible, c'est-à-dire soyons près de la vie primordiale. Mais toute expérience du Rien ne conduit-elle pas au désespoir, puisque cette expérience extrême qui dévoile les tensions chaotiques de la vie, révèle aussi le néant de l'existence? Peut-on être serein et désespéré face à cette découverte des profondeurs? Deux attitudes se dessinent clairement: 1) celui qui désespère parce qu'il a conscience de la réalité abyssale et par conséquent des illusions du réel à perdre. 2) Et celui qui demeure serein parce qu'il perçoit les forces vitales chaotiques et tente de s'adapter à elles. Comme le surfeur, qui connaît la force et le danger des immenses vagues de la mer, l'homme serein s'y risque parce qu'il sait qu'elles peuvent le porter merveilleusement, tout comme le projeter mortellement sur les rochers de la côte. L'être serein demeure dangereusement, mais intensément lucide au cœur des forces démoniques.

Ne faut-il pas être un peu fou pour se jeter volontairement dans le chaos ou pour désirer vivre un ravissement dans l'existence? L'éclair ne dure pas; il transporte l'homme à un point de non retour, où l'équilibre est impossible à rétablir. Est fou celui qui est happé par le chaos et qui ne perçoit plus ensuite la concrétude. Mais peut-on être un fou heureux, un fou sans les dépressions et la crainte d'avoir à affronter un moment de lucidité dans sa folie? Cioran désire bien être un foudroyé, qui serait uniquement enveloppé d'une folie lumineuse, paradisiaque; il désire vivre l'intensité extatique, transfigurative sans prendre conscience du désenchantement, de la baisse de tension consécutive à ce ravissement.

Cioran décrit comment certains des hommes perçoivent lucidement la réalité. Aucune illusion d'une autre vie n'est possible ici-bas et encore moins dans un au-delà. Cette vie leur suffit déjà amplement. Par contre, il s'intéresse grandement aux tentatives d'esquiver la réalité, c'est-à-dire de la transcender.

Résumons-nous. Le premier élément de l'horizon cioranien développé ici est celui du Rien originel, qui appelle parfois dans le temps à une conversion. L'intensité de cette expérience-limite dévoile d'une part les forces chaotiques de la vie et d'autre part le néant de la vie ordinaire. Une fois le ressort étiré jusqu'à l'extase, il n'y a plus d'équilibre possible. L'homme se forge des planches de salut, qui ne lui permettent plus de voir le réel tel quel. Cioran décrit les illusions à perdre; il appelle à la lucidité 
pour se purger de tout et vivre de manière sensible le vertige de l'infini : un infini parfois ténébreux, parfois lumineux. Le premier est conscient de son néant, c'est-à-dire de la présence mortifère portant la vie vers la désintégration, vers le néant. Le second est désir d'une expérience extrêmement lumineuse, extase, plénitude, excès. Tentons maintenant de mieux situer ce que Cioran entend par le Rien.

\section{Compréhensions du Rien}

Mon étude ne pourra être exhaustive, car même si plusieurs fragments usent des termes qui nous intéressent, ils ne sont pas toujours suffisamment encadrés pour nous permettre un développement pertinent de la constellation. Je retiens deux contextes qui peuvent contribuer à mieux cerner la compréhension cioranienne du Rien : un premier associe le Rien et le néant à la mort et un autre le met en relation avec Dieu.

\subsection{La mort et le Rien}

Nous avons plus haut situé le néant dans l'ordinaire et le Rien dans le radical, mais qu'en est-t-il lorsque la mort nous conduit à la frontière de l'existence et de la non-existence, à la frontière de l'histoire et de sa fin, à la frontière du temps et de son anéantissement? Le texte intitulé Sur la mort précise le lien entre le Rien et le néant. Ici, la distinction entre vie ordinaire et vie essentielle est toujours présente. Dans la première, l'aventure, la recherche spirituelle, l'élan indéfini vers l'infini, bref la tentation d'accéder à une autre réalité apparaît comme superficielle, illusoire. Dans la seconde, on ne quête ni ne recherche sérieusement, mais on est soumis à cette folle tension qui élève au plan de l'éternité. La première est une escalade laborieuse mais vaine, la seconde une pression et une élévation extatique.

Mais comment comprendre la mort? Comme un terme à la vie ou comme son compagnon de tous les jours? Cioran opte pour la seconde représentation qui blesse et dérange les bien-portants. Pour lui, impossible d'oublier la captivité de la vie par la mort, impossible d'occulter la présence de la mort dans la vie. Plusieurs assurément refoulent cette macabre réalité; ils vivent normalement en se disant qu'un jour la mort surviendra. Pour l'écrivain roumain, au contraire, vivre est une longue agonie, où chaque pas dans la vie est un pas dans la mort, où le souvenir de la mort est un rappel du néant. Et cette conscience n'origine pas d'une lecture spéculative superficielle; elle sourd de l'angoisse et des larmes; elle est testée dans le regard blanchi par la peur et dans le cri sauvage du désespoir. Vivre avec cette conscience de la mort rompt le charme des illusions possibles, des projets finalistes et des consolations théologiques. Cette conscience a saveur d'anéantissement, de néant. Il est certain que 
ce mélange de vie et de mort démasque les prétentions de l'existence : elle est nulle, insignifiante. Ne serait-ce pas dans cette conscience que se manifeste le caractère démonique de la vie, c'est-à-dire cette effervescence intérieure à la fois créatrice et destructrice? Y a-t-il extase démonique à travers le tissu du réel, Cioran parle alors de la vie comme d'une longue agonie. Y a-t-il extase lumineuse, créatrice, Cioran parle alors d'un au-delà ou d'un en-deça de la vie, d'une vie avec un grand $V$.

Selon Cioran, seuls les gens affectés par la maladie ou par des états dépressifs peuvent parler de manière crédible de l'immanence de la mort dans la vie. Cette irruption de la mort dans la structure même de la vie introduit, selon lui, implicitement le néant dans l'élaboration de l'être :

L'irruption de la mort dans la structure même de la vie introduit implicitement le néant dans l'élaboration de l'être. De même que la mort est inconcevable sans le néant, de même la vie est inconcevable sans un principe de négativité. L'implication du néant dans l'idée de la mort se lit dans la peur qu'on en a, qui n'est autre que l'appréhension du Rien 20 .

Vie et mort ne sont-ils qu'un processus, processus marqué pour la mort par le néant, mode de vie cheminant vers l'anéantissement et processus marqué pour la vie d'une sortie du chaos, d'un dépassement du principe de négativité? Ne semble-t-il pas alors contradictoire d'opposer le couple mort-néant au couple vie-principe de négativité? Dans un horizon présupposant la primauté de la vie sur la mort et de l'être sur le néant ceci étonne grandement. Mais inversons l'horizon et accordons la primauté à la mort sur la vie et au néant sur l'être. Ne faut-il pas alors effectivement un principe de négativité pour rendre la vie possible?

Selon Cioran, la peur de la mort révèle la peur du Rien, c'est-à-dire la peur de la victoire du non-être sur l'être. Ce mélange de vie et de mort constitue l'existence, une existence très souvent tentée de masquer la mort et de la repousser à la fin au lieu d'accepter de voir l'homme pétri par elle dans le temps et de concevoir la mort comme un cheminement vers le néant, ultimement le Rien. Ainsi, on exclut les semblants de salut (naïveté, inconscience), les illusions de salut (vie éternelle, dépassement de la mortalité) et les saluts issus du néant. De quoi sommes-nous sauvés si l'existence aboutit dans le Rien? Ce dernier est-il un néant hors-temps, l'aboutissement du néant? Le Rien représente-t-il simplement un conceptlimite de l'originel, du potentiel, de l'être hors temps? Mais s'il n'y a de salut ni dans l'existence, ni dans le néant, donc ni dans la sortie, le 
déploiement, la manifestation du réel et ni dans la disparition, la décroissance, la disparition du réel, y a-t-il salut dans le Rien? Mais de quoi serions-nous sauvés, sinon de l'existence?

\subsection{Dieu et le Rien}

Dieu est souvent associé au rien (sans majuscule) ou au néant. Il appert alors n'être qu'un vocable insignifiant. Mais même s'il nie être croyant ou chrétien, Cioran ne garde-t-il pas profondément ancré dans sa mémoire le récit de l'Autre, le mystère du Rien? Si Dieu existe, il le dénonce et le maudit pour avoir placé l'homme dans un tel pétrin. Veut-il alors fracasser nos représentations, nos anthropomorphismes et nos croyances à propos du mystère divin? Bien que Cioran soit sans Dieu, il envie ceux qui le vénèrent. Mais pour lui, Dieu est un mot pour nommer le rien du monde. Le néant est parfois la forme existentielle du Rien. Il est la poussière de la création, la glu de la vie et il ne cesse d'engluer toute l'existence. Se tenir debout dans cette existence néantisée, c'est le destin de cet amas de glu.

Dans Des larmes et des saints, on retrouve les termes du néant et du rien associés au mot Dieu. $\mathrm{Si}$, par exemple, le Dieu d'antan pouvait contenir le monde et l'accueillir en son néant, il semble qu'aujourd'hui "le rien du monde soit privé de souffle divin 21 ". Le monde actuel est-il tout simplement plus lucide qu'autrefois? Un peu plus loin, Cioran affirme que «le champ visuel du cœur [est] le monde, plus Dieu, plus le néant. C'est-à-dire tout 22 ". Le Dieu dont il est ici question est selon nous le Dieu de la totalité. Et quelques pages plus loin, "Dieu, rival du Rien, apparaît comme une dernière tentation 23 ". Mais ne s'agit-il pas encore une fois du Dieu de la totalité? Le Rien ne pourrait-il pas être l'infini? Il écrit encore un peu plus loin à propos de Dieu et du rien comment ils sont près l'un de l'autre : "Tout est rien - telle est la révélation initiale des couvents. Ainsi commence la mystique. Entre le rien et Dieu, il y a moins qu'un pas, car Dieu est l'expression positive du rien ${ }^{24}$ ». Ici, la compréhension du Dieu de la totalité se confirme. Mais Dieu n'est-il que la totalité? Il semble bien que chez Cioran, il faille répondre par l'affirmative.

Dieu est chez lui situé sous l'horizon existentiel et voisine donc le néant; Dieu apparaît comme un alibi pour «masquer » la folie ou comme

Emil CIORAN, « Des larmes et des saints ", dans CEuvres, p. 290. Idem.

Ibid., p. 295.

Ibid., p. 304. 
une excuse à l'égarement: "Dieu? Une démence admise, officielle 25 ". "La religion, dans ce sens, est un sourire qui plane sur un non-sens général, comme un parfum final sur une onde de néant ${ }^{26}$ ". Ailleurs, les attributs de Dieu apparaissent comme la simple projection de nos humeurs : "nous le craignons comme un monstre et l'écrasons comme un insecte; nous l'idolâtrons: il est l'Être; le repoussons : il est le Rien ${ }^{27}$ ". Une fois bien débarrassée de ses attributs, la créature « ira rejoindre dans le néant sa plus haute invention : son créateur 28 ". On voit ici comment Cioran conçoit Dieu: « Dieu : fruit de l'inquiétude de nos entrailles et du gargouillement de nos idées... Seule l'aspiration au Vide nous préserve de cet exercice de souillure qu'est l'acte de croire ${ }^{29}$ ». Qu'est-ce à dire?

La mystique oscille entre la passion de l'extase et l'horreur du vide. On ne peut connaître l'une sans avoir connu l'autre. [...] Une conscience dépossédée de toutes les images est la condition indispensable de l'état d'extase et de l'expérience du vide. On ne voit plus rien en dehors du rien, et ce rien est tout. L'extase est une présence totale sans objet, un vide plein. Un frisson traverse le néant, une invasion d'être dans l'absence absolue. Le vide est la condition de l'extase, de même que l'extase est la condition du vide ${ }^{30}$ ».

Cette définition de la mystique, s'appuyant sur la tension entre l'extase et le vide, exclut Dieu comme objet, comme totalité. Ne pourraiton pas alors situer la dimension mystique, extatique, comme la dimension où l'absolu fait irruption dans l'histoire ${ }^{31}$, dimension dépassant celle de l'existence, du néant et ultimement de Dieu?

Malgré l'âpre critique de Dieu et de la religion, remarquons comment Cioran cherche à articuler un au-delà de l'existence par le biais de la mystique. Le Dieu critiqué est celui de la totalité qui fréquente le néant de l'existence. L'absolu, la présence, le Rien fait irruption au cœur de l'existence et lui révèle son néant. Et s'il faisait irruption au cœur du vide, lui révèlerait-il son être? Ces dernières remarques corroborent l'intérêt de Cioran, non pas uniquement pour la pré-existence, mais aussi pour le

Ibid., p. 306.

26 lbid., p. 309.

27 Emil CloRan, "Précis de décomposition ", dans EEuvres, p. 701.

28 Id.

29 Ibid., p. 701-702.

30 Emil CIORAN, « Des larmes et des saints », dans Furres, p. 305-306.

31 Voir ibid., p. 306. 
temps présent, où dans l'instant le vide est rempli par la présence de l'éternel maintenant qui dépasse infiniment le temps.

\section{Conclusion}

«Osciller entre l'Apparence et le Rien, entre la forme trompeuse et son absence : vibration entre deux irréalités ${ }^{32}$ ".

Cioran voulait-il simplement écrire pour se libérer de la peur de faire comme tout le monde : désirer et rêver, se forger des mythes et des projets, tenter de résoudre les incompréhensions, le mal de l'existence et espérer malgré tout et en dépit de tout? Ou voulait-il jeter des mots à la tête du Rien pour lui permettre d'être? Ne voulait-il pas simplement tenir bon dans son lâcher prise, difficile et exigeante entreprise pour quelqu'un qui doit se poser ou mieux, osciller dans la vacuité. La vacuité apparaît à la fin de ce parcours comme un lieu prometteur pour Cioran.

Ne pourrions-nous pas comprendre son œuvre comme une meta odos, comme un chemin vers le Rien, au long duquel les manèges de la raison, de l'esprit et de la conscience sont détraqués. Ils se brisent au contact (ou à la prise de conscience qui se développe au long de ce parcours) du réel brut, du rien, du néant qui cerne l'homme. Ils sont démantelés si l'homme, en plus de percer et d'évacuer son inanité, fait l'expérience du Rien, expérience saisissante, exaltante, enthousiasmante d'un non-dit, d'un inter-dit, expérience de l'éternel présent. Les termes varient, mais Cioran ne module-t-il pas sans cesse un thème unique : celui du néant d'être et du Rien? L'écriture organisée en fragments, dont la fonction principale est de rendre plus supportables l'ennui et le désespoir, ne conduit pas méthodiquement au Rien. Elle deviendrait alors religieuse et tomberait elle-même sous la critique cioranienne. L'écriture déshabille la réalité sans lui proposer de nouveaux voiles. Elle reste nue, s'apprête à sauter dans le Vide, faille au creux de l'existence, que Cioran ne désire qu'agrandir.

La lecture cioranienne du néant et du Rien, le néant habitant l'existence et le Rien la dépassant, est une lecture à rebours par rapport à la tradition occidentale, car elle dénonce l'hérésie affirmant la victoire de l'être sur le non-être, le dépassement réel du mal par le bien ou encore la possibilité d'être sauvé et de vivre d'une vie dite éternelle. Cioran bouscule ce schème sécurisant et décrit la pesanteur de l'être, la fatigue de l'existence, les manigances crucifiantes du souffrant pour anesthésier le mal; la vie, à part ses soubresauts d'éternité, est dure et souffrante. 
Le Rien ne sauve pas, mais n'évoque-t-il pas le bouillonnement de la vie? Ne serait-il pas présupposé au Dieu créateur, principe chaotique débarrassé de la raison et de l'ordre? Originel, le Rien ne s'apparenteraitil pas au deus absconditus? Le Rien, un Dieu au-delà de Dieu? Un Dieu, source de vie intense et chaotique, un Dieu indicible et irréductible. Pourquoi pas? Et lorsque plus tard dans ses écrits le Rien semble perdre son caractère chaotique et vitalisant, Cioran ne le transforme-t-il pas en un concept-limite, concept tout aussi capable de rendre compte autrement de l'avènement de Dieu, une fois l'attitude d'évidement endossée par l'homme?

Et si le théologien peut endosser, dans une large part, la critique des illusions et des mots tronqués, le théologien chrétien, parce qu'il le gère autrement, ne s'opposera pas fondamentalement au néant de l'existence. Ce néant est pour lui dépassé par la lumière du Rien. Chez Cioran, cette lumière se rencontre peut-être une fois évidée du néant de l'existence. Le refus ou l'intégration du néant semble indiquer une différence fondamentale entre Cioran et le théologien. L'écrivain remet aussi foncièrement en question la construction théologique sécurisante, lorsqu'il concentre ses efforts à démasquer les fausses apparences de l'existence. Cioran illustre existentiellement ce que le théologien ne peut plus escamoter, s'il désire rencontrer le monde d'aujourd'hui. Sans ce séjour au creux du néant, on ne peut penser témoigner et intelliger le Dieu du Christ, on ne peut penser rencontrer la lumière du Rien. 